\title{
Zur Jodierung von Verbindungen des Eiweißgebietes.
}

\author{
Von
}

Herm. Pauly.

(Der Redaktion zugegangen am 28. November 1911.)

Tyrosin und Histidin verhalten sich bei der «Kern $*-J o d i e-$ rung, d. h. bei der Bindung von Jod an Kohlenstoff ihres Moleküls den Stammkörpern, von denen sie sich als Glykokollverbindungen ableiten, vollkommen analog. Tyrosin jodiert sich zu der dem 3,5-Dijodkresol analog zusammengesetzten *Jodgorgosäure», Histidine schließen sich, wie ich gezeigt habe, ${ }^{1}$ ) den Imidazolen an.

Man darf somit, wenn festgestellt ist, daß Skatol nicht jodierbar ist, ${ }^{2}$ ) erwarten, daß auch dessen Glykokollderivat, das Tryptophan, kein Jod aufnehmen kann, wie schon früher hervorgehoben wurde. ${ }^{3}$ )

Bei dieser Gelegenheit ist zunächst nochmals ${ }^{4}$ ) darauf hinzuweisen, daß im Hinblick auf die relative Festigkeit, mit der das Jod in natürlichen Jodproteinen haftet, zur Entscheidung der Frage, ob Aminosäuren, Eiweißkörper usw. Jod binden können oder nicht, es nicht genügt, überhaupt nur jodhaltige Produkte erhalten zu haben. Wohl jeder basischen Stickstoff enthaltende organische Körper liefert farbige perjodidartige und, wenn der Stickstoff noch Wasserstoff trägt, auch N-jodierte Verbindungen. In allen diesen ist das Jod aber mehr oder weniger lose gebunden. ${ }^{5}$ ) Als äußere Kriterien festgebundenen

1) Ber. d. Deutsch. chem. Ges., Bd. 43, S. 2243 (1910).

2) Pauly und Gundermann, Ber. d. Deutsch. chem. Ges., Bd. 41, S. 3999 (1909). - Oswald, Diese Zeitschrift, Bd. 73, S. 128 (1911).

s) l. c.

4) $1 . \mathrm{c}$.

5) Außer dem von mir durch äußerst vorsichtige Jodierung von Sturin erhaltenen farblosen Jodsturin dürften alle bisher auf künstlichem Wege erhaltenen Jodproteine den größten Teil ihres Jods in dieser lockeren Form enthalten, da sie durchwegs mit Jod in zu großen Konzentrationen dargestellt sind und braune Pulver (siehe Cohnheim, Chemie der Eiweißkörper, 3. Aufl., S. 138) darstellen. 
Jods betrachte ich gemeinhin, daß die jodierten Produkte 1 . in frisch dargestelltem Zustande rein weiß sind und 2. daß sie bei kürzerer Einwirkung kalter verdünnter schwefliger Säure (Thiosulfat genügt nicht) ihr Jod nicht verlieren, also z. B. nicht bei der Ausfällung mit dieser Säure aus ihren Salzen bei gewöhnlicher Temperatur.

Wenn daher Herr Neuberg ${ }^{1}$ ) aus Jod und Tryptophan braune amorphe Pulver präpariert, die er Jodtryptophane nennt, so ist damit für die Frage des Jodbindungsvermögens in dem oben gemeinten Sinne nichts gewonnen. Um so weniger, wenn die Stoffe in Gegenwart von Alkali erhalten wurden, weil man dann bei der freien Aminosäure mit großer Sicherheit einen gleichzeitigen Abbau des Alaninrestes durch die alkalische Hypohalogenitlösung zu erwarten hat.

Ich selbst konnte bisher Monobenzoyl-tryptophan, das in der üblichen Weise nach dem Verfahren von SchottenBaumann aus natürlichem Tryptophan gewonnen war, weder mit noch ohne Alkali jodieren, d. h. «kernjodieren». Allerdings wäre denkbar, daß die Jodierung hier deswegen ausgeblieben sei, weil das Benzoyl nicht am Alanin-, sondern am Indolstickstoff hinge. Nach den bisherigen Erfahrungen über die Benzoylierung derartiger Körper ist dies jedoch wenig wahrscheinlich. Auch spricht der Umstand, daß die Benzoylverbindung Skatolreaktionen zeigt, nicht gerade zugunsten dieser Annahme. (Leider mußte diese Frage aus Mangel an dem sehr kostbaren Material noch offen bleiben.)

Auch freies Tryptophan kann nach $\mathrm{Oswald}^{2}$ ) nicht in Jodierungsprodukte übergeführt werden.

1) Biochemische Zeitschrift, Bd. 6, S. 276 (1907).

2) 1. c. 